\title{
A NOTE ON ASYMPTOTIC HELIX AND QUANTUM MECHANICAL STRUCTURE
}

\section{PARTHA GUHA}

Received 1 December 2002 and in revised form 2 January 2003

Using the formulation of a moving curve, we demonstrate that an asymptotic helix goes over to the linear time-dependent Schrödinger equation as shown by Dmitriyev (2002).

2000 Mathematics Subject Classification: 81P99, 76B47, 76B25, 35 Q55.

1. Introduction. Here we consider unbounded, inviscid, and incompressible fluid flows. In the absence of external force, the motion of such fluid with unit density is described by the Euler equations

$$
\partial_{t} \mathbf{u}+\mathbf{u} \cdot \nabla \mathbf{u}=-\nabla p, \quad \nabla \cdot \mathbf{u}=0
$$

where $\mathbf{u}(\mathbf{x}, t)$ is the velocity, $\mathbf{x}=\left(x_{1}, x_{2}, x_{3}\right)$ is the position, $p$ is the pressure, and $\nabla$ is the differentiation vector. The curl of the velocity field

$$
\omega=\nabla \times \mathbf{u}
$$

is the vorticity. The velocity $\mathbf{u}(\mathbf{x})$ can be determined from the vorticity $\omega(\mathbf{x})$ through the Biot-Savart law:

$$
\mathbf{u}(\mathbf{x})=-\frac{1}{4 \pi} \int \frac{\left(\mathbf{x}-\mathbf{x}^{\prime}\right) \times \omega\left(\mathbf{x}^{\prime}\right)}{\left|\mathbf{x}-\mathbf{x}^{\prime}\right|^{3}} d \mathbf{x}^{\prime} .
$$

If the vorticity is concentrated on a single thin filament $C$ of circulation $\Gamma$, equation (1.3) becomes

$$
\mathbf{u}(\mathbf{x})=-\frac{\Gamma}{4 \pi} \int_{C} \frac{\left(\mathbf{x}-\mathbf{x}^{\prime}\right) \times d \mathbf{l}\left(\mathbf{x}^{\prime}\right)}{\left|\mathbf{x}-\mathbf{x}^{\prime}\right|^{3}} d \mathbf{x}^{\prime}
$$

If one computes the self-induced motion of the filament by evaluating the velocity from (1.4) on the filament itself, then the result is logarithmically infinite if the filament is curved and zero if it is straight. So the self-induced motion occurs only for curved filaments. We must focus our attention on a very slender vortex filament.

One can avoid singularity in (1.4) by simply ignoring the nonlocal contribution of the filament and replace the Biot-Savart law by a velocity expression 
that depends only on the local curvature of the vortex filament. If a vortex filament is described by $\mathbf{r}(s)$, where $s$ is an arc length parameter measured along the filament, then $\mathbf{r}(s)$ is the position vector. Let $\mathbf{t}, \mathbf{n}$, and $\mathbf{b}$ denote the unit tangent, normal, and binormal vectors, respectively.

Hence this leads to the local induction approximation, this is,

$$
\frac{\partial \mathbf{r}}{\partial t}=\kappa \mathbf{b}=\mathbf{t} \times \frac{\partial \mathbf{t}}{\partial s}
$$

where $\kappa$ is the curvature. Differentiating both sides with respect to arc length $s$ yields the Heisenberg spin chain equation in terms of tangent vector:

$$
\frac{\partial \mathbf{t}}{\partial t}=\kappa \mathbf{b}=\mathbf{t} \times \frac{\partial^{2} \mathbf{t}}{\partial s^{2}}
$$

In this paper, we investigate the relation between filament equation and Schrödinger equation of quantum mechanics. We consider the (stationary) Schrödinger equation

$$
-\frac{\hbar^{2}}{2 m} \psi_{X X}+V(X) \psi=E \psi
$$

This equation can easily be transformed into

$$
\psi_{x x}-v(x) \psi=-E \psi
$$

where $\iota=\hbar / \sqrt{2 m}$ and $\iota \partial_{X}=\partial_{x}$. Similarly, we can scale the time-dependent Schrödinger equation

$$
-i \hbar \psi_{\tau}=-\frac{\hbar^{2}}{2 m} \psi_{X X}+V(X) \psi
$$

We scale

$$
\tau \longrightarrow T=-\left(\frac{1}{\sqrt{2 m}}\right) \tau
$$

hence (1.9) becomes

$$
-i \iota \psi_{T}=-\iota^{2} \psi_{X X}+V \psi
$$

Then for $\iota x=X$ and $i \iota t=T$, one has

$$
\psi_{t}=\psi_{x x}-v \psi
$$

It is well known that under the connection between the Hasimoto vortex solitons [8] and the nonlinear Schrödinger equation (NLSE), the basis in the FrenetSerret frame $(t, n, b)$ is associated to certain quantities in NLS equations. Subsequently, Lamb [9] demonstrated that various integrable systems could also 
be associated with the motion of a curve. In recent years, there has been a tremendous growth of interest in the applications of the Frenet-Serret equations for a curve to various contexts of integrable systems [3, 6, 7, 10, 11, 12].

Balakrishnan and Dandoloff [2] have shown that the time-dependent Schrödinger equation for a particle in a potential $V(s, u), u$ denoting time, can be interpreted geometrically as a moving curve whose Fermi-Walker phase density is given by $-(\partial V / \partial s)$. In an extremely interesting paper, Dmitriyev [4] showed that the small amplitude to thread ratio helical configuration of a vortex filament in the ideal fluid behaves exactly as de Broglie wave. In this paper, using the formulation of a moving curve, we give a direct proof of how an asymptotic helix goes over to the linear time-dependent Schrödinger equation as shown by Dmitriyev.

2. Frenet-Serret frame. We consider a space curve $\gamma(s)$, parametrized by arc length $s ; \kappa$ and $\tau$ are the curvature and the torsion of the space curve. We denote by $\mathbf{t}$ the unit tangent vector to this curve and by $\mathbf{n}$ and $\mathbf{b}$ its principle normal and binormal, respectively. Then the Frenet frame $\{\mathbf{t}, \mathbf{n}, \mathbf{b}\}$ forms a moving triad of the curve and they form a right-handed system of axes, that is,

$$
\mathbf{b}=\mathbf{t} \times \mathbf{n} .
$$

A space curve embedded in $3 D$ is described using the usual Frenet-Serret equations (see, e.g., [5]):

$$
\left(\begin{array}{l}
\mathbf{t} \\
\mathbf{n} \\
\mathbf{b}
\end{array}\right)_{S}=\left(\begin{array}{ccc}
0 & \kappa & 0 \\
-\kappa & 0 & \tau \\
0 & \tau & 0
\end{array}\right)\left(\begin{array}{l}
\mathbf{t} \\
\mathbf{n} \\
\mathbf{b}
\end{array}\right)
$$

and these underlie central investigations in the theory of space curves. From the equation, $\kappa$ and $\tau$ are given by

$$
\begin{gathered}
\kappa^{2}=\mathbf{t}_{s} \cdot \mathbf{t}_{s}, \\
\boldsymbol{T}=\frac{\mathbf{t} \cdot\left(\mathbf{t}_{s} \times \mathbf{t}_{s S}\right)}{\boldsymbol{\kappa}^{2}} .
\end{gathered}
$$

If we introduce the Darboux vector ( $s$-angular velocity of Frenet frame)

$$
\Omega=\tau \mathbf{t}+\kappa \mathbf{b}
$$

then the Frenet-Serret equations may be written as

$$
\mathbf{t}_{s}=\Omega \times \mathbf{t}, \quad \mathbf{n}_{s}=\Omega \times \mathbf{n}, \quad \mathbf{b}_{s}=\Omega \times \mathbf{b} .
$$


If the curve moves with time $u$, then all quantities in (2.2) become functions of both $s$ and $u$, and the time evolution is given by

$$
\left(\begin{array}{l}
\mathbf{t} \\
\mathbf{n} \\
\mathbf{b}
\end{array}\right)_{u}=\left(\begin{array}{ccc}
0 & f & g \\
-f & 0 & h \\
-g & -h & 0
\end{array}\right)\left(\begin{array}{l}
\mathbf{t} \\
\mathbf{n} \\
\mathbf{b}
\end{array}\right)
$$

This set of equations may be written as

$$
\mathbf{t}_{s}=\tilde{\Omega} \times \mathbf{t}, \quad \mathbf{n}_{s}=\tilde{\Omega} \times \mathbf{n}, \quad \mathbf{b}_{s}=\tilde{\Omega} \times \mathbf{b},
$$

where $\tilde{\Omega}$ is another Darboux vector given by

$$
\tilde{\Omega}=h \mathbf{t}+g \mathbf{n}+f \mathbf{b}
$$

The compatibility condition of (2.2) and (2.6) leads to

$$
\begin{aligned}
& \frac{\partial \kappa}{\partial u}-\frac{\partial f}{\partial s}+\tau g=0 \\
& \frac{\partial \tau}{\partial u}-\frac{\partial h}{\partial s}-\kappa g=0 \\
& \frac{\partial g}{\partial s}-\kappa h+\tau f=0 .
\end{aligned}
$$

2.1. Lamb formalism of moving curves. The Frenet-Serret equations can be combined to give one complex and one real equations [7]

$$
\begin{gathered}
(\mathbf{n}+i \mathbf{b})_{s}+i \tau(\mathbf{n}+i \mathbf{b})=-\kappa \mathbf{t}, \\
\mathbf{t}_{s}=\kappa \mathbf{n} .
\end{gathered}
$$

Following Lamb, we introduce a complex quantity

$$
\mathbf{N}=(\mathbf{n}+i \mathbf{b}) \exp \left[i \int_{-\infty}^{s} d s^{\prime} \boldsymbol{\tau}\right]
$$

DEFINITION 2.1. The Hasimoto transformation is a map which assigns to a space curve a complex curvature function via the formula

$$
\gamma \longrightarrow \mathscr{H}(\gamma), \quad q=\kappa(s) \exp \left[i \int_{-\infty}^{s} d s^{\prime} \tau\right] .
$$

The image of the Hasimoto map is defined up to an arbitrary constant phase $\phi$.

The Frenet-Serret equations in terms of $\left\{\mathbf{t}, \mathbf{N}, \mathbf{N}^{*}\right\}$ are

$$
\begin{gathered}
\mathbf{N}_{s}=-q \mathbf{t}, \\
\mathbf{t}_{s}=\frac{1}{2}\left(q^{*} \mathbf{N}+q \mathbf{N}^{*}\right) .
\end{gathered}
$$


LEMMA 2.2. The new Frenet frame $\left\{\mathbf{t}, \mathbf{N}, \mathbf{N}^{*}\right\}$ satisfies the following conditions:

$$
\begin{gathered}
\mathbf{N} \cdot \mathbf{t}=\mathbf{N}^{*} \cdot \mathbf{t}=\mathbf{N} \cdot \mathbf{N}=0 \mathbf{N} \cdot \mathbf{N}^{*}=2, \\
\mathbf{N} \times \mathbf{t}=i \mathbf{N}, \quad \mathbf{N}^{*} \times \mathbf{t}=-i \mathbf{N}^{*}, \\
\mathbf{N}^{*} \times \mathbf{N}=2 i \mathbf{t} .
\end{gathered}
$$

Then this complex basis $\left\{\mathbf{t}, \mathbf{N}, \mathbf{N}^{*}\right\}$ can be identified with $\mathrm{SO}(3)$, where $\mathbf{t}$ is an element of the unit sphere and we regard $\left(\mathbf{N}, \mathbf{N}^{*}\right)$ as a positive orthonormal frame of the tangent space of $S^{2}$ at $\mathbf{t}$.

2.2. Equations of curve. The derivatives of $\mathbf{N}$ and $\mathbf{t}$ with respect to $u$ may be written, in general, as

$$
\begin{aligned}
\mathbf{N}_{u} & =\epsilon \mathbf{N}+\mu \mathbf{N}^{*}+\gamma \mathbf{t}, \\
\mathbf{t}_{u} & =\lambda \mathbf{N}+\pi \mathbf{N}^{*}+\nu \mathbf{t} .
\end{aligned}
$$

Using (2.13) and the compatibility conditions of $\mathbf{t}_{u s}=\mathbf{t}_{s u}$, these equations boil down to

$$
\begin{aligned}
\mathbf{N}_{u} & =i C \mathbf{N}+\gamma \mathbf{t}, \\
\mathbf{t}_{u} & =-\frac{1}{2}\left(\gamma^{*} N+\gamma \mathbf{N}^{*}\right),
\end{aligned}
$$

where $C=C(s, u)$ is a real function.

Using the compatibility condition $\mathbf{N}_{u s}=\mathbf{N}_{s u}$, we obtain one complex and one real equations

$$
\begin{gathered}
q_{u}+\gamma_{s}-i C q=0, \\
C_{s}=\frac{i}{2}\left(\gamma q^{*}-\gamma^{*} q\right) .
\end{gathered}
$$

In Section 3, we will see that the integrodifferential equation

$$
q_{u}+\gamma_{s}+\frac{q}{2} \int_{-\infty}^{s}\left(\gamma q^{*}-\gamma^{*} q\right) d s^{\prime}=0
$$

connects the moving space curve equations and other evolutionary systems.

Since $\mathbf{t} \cdot \mathbf{t}_{u}=0$, we may assume that

$$
\mathbf{t}_{u}=f \mathbf{n}+g \mathbf{b} .
$$

Then from

$$
\mathbf{t}_{u}=-\frac{1}{2} \gamma^{*}(\mathbf{n}+i \mathbf{b}) \exp \left[i \int_{-\infty}^{s} d s^{\prime} \boldsymbol{\tau}\right]+\gamma(\mathbf{n}-i \mathbf{b}) \exp \left[-i \int_{-\infty}^{s} d s^{\prime} \boldsymbol{\tau}\right]
$$


we obtain

$$
\gamma=-(f+i g) \exp \left[i \int_{-\infty}^{s} d s^{\prime} \tau\right] .
$$

Proposition 2.3 (zero-curvature equation). The compatibility conditions of

$$
\begin{aligned}
& \frac{\partial \phi}{\partial s}=\frac{1}{2}\left(\begin{array}{cc}
0 & -q \\
q^{*} & 0
\end{array}\right) \phi, \\
& \frac{\partial \phi}{\partial u}=\frac{1}{2}\left(\begin{array}{cc}
i C & \gamma \\
-\gamma^{*} & -i C
\end{array}\right) \phi
\end{aligned}
$$

describe the motion of moving curves.

PROOF. By direct computation, the off-diagonal term satisfies (2.18) and the diagonal term yields (2.19).

\subsection{Examples}

EXAMPLE 2.4. We first consider the Belavin-Polyakov equation $\mathbf{t}_{u}=-\mathbf{t} \times \mathbf{t}_{s}$. In this case, $f=0$ and $g=-k$. Hence $\gamma=i q$ and $C=-\int_{\infty}^{s}|q|^{2} d s^{\prime}$. Thus the equation of motion is

$$
q_{u}-i q_{s}-i q \int_{\infty}^{s}|q|^{2} d s^{\prime}
$$

This equation was introduced in [1]. The real part of this equation coincides with the elliptic Liouville equation.

EXAMPLE 2.5. We consider the Heisenberg spin chain equation $\mathbf{t}_{u}=\mathbf{t} \times \mathbf{t}_{s \mathcal{s}}$. It is easy to see that

$$
\mathbf{t} \times \mathbf{t}_{s s}=\kappa_{s} \mathbf{b}-\kappa \tau \mathbf{n}
$$

Thus we can identify $f=-\kappa \tau, g=\kappa_{s}$, and

$$
\gamma=-\left(-\kappa \tau+i \kappa_{s}\right) \exp \left[-\int_{-\infty}^{s} d s^{\prime} \tau\right]=-i q_{s} .
$$

Also, $C_{s}=(1 / 2)|q|_{s}^{2}$. Thus the equation of motion becomes NLSE

$$
i q_{u}+q_{s s}+\frac{1}{2}|q|^{2} q=0 .
$$

3. Connection to quantum mechanics. We consider an approximation of (2.18). Suppose that $g \ll f$, then

$$
\gamma \sim f \exp \left[i \int_{-\infty}^{s} \tau d s^{\prime}\right] .
$$


It is easy to see that, for $g \ll f$,

$$
C_{s}=\frac{i}{2}[(f+i g) \kappa-(f-i g) \kappa] \longrightarrow 0 .
$$

Thus (2.17) becomes

$$
q_{u}+\gamma_{s}=0 \text {. }
$$

Proposition 3.1. The Hasimoto mapping from the Heisenberg spin chain equation yields the time-dependent Schrödinger equation for $\kappa \ll \tau$.

Proof. The proof is given by direct computation.

In the event, $\kappa \ll \tau$ tends to an asymptotic helix. This is the humped helix approximated by the wave packet, as shown by Dmitriyev [4].

We consider the solutions of the Schrödinger equation

$$
q=a\left[\cos \left(\tau s-\tau^{2} u\right)+i \sin \left(\tau s-\tau^{2} u\right)\right] \equiv a \exp \left[i\left(\tau s-\tau^{2} u\right)\right],
$$

and $\phi=\psi_{1}(s, u)+i \psi_{2}(s, u)$, then this denotes a helix rotating counterclockwise around the $s$-axis with the constant angular velocity $\omega=\tau^{2}$.

3.1. Schrödinger equation with potential and geometric phase. We consider a generalized Hasimoto map

$$
q=\kappa e^{i \int_{-\infty}^{s} \tau d s^{\prime}+i \lambda u}, \quad \gamma=-(f+i g) e^{i \int_{-\infty}^{s} \tau d s^{\prime}+i \lambda u},
$$

where $\lambda$ is some parameter. Then the zero-curvature equation becomes

$$
q_{u}+\gamma_{s}-i C q+i \lambda \gamma=0
$$

This results from the compatibility condition of

$$
\frac{\partial \phi}{\partial s}=\frac{1}{2}\left(\begin{array}{cc}
i \lambda & -q \\
q^{*} & -i \lambda
\end{array}\right) \phi, \quad \frac{\partial \phi}{\partial u}=\frac{1}{2}\left(\begin{array}{cc}
i C & \gamma \\
-\gamma^{*} & -i C
\end{array}\right) \phi .
$$

So the Schrödinger equation becomes

$$
i q_{u}+q_{s s}+\lambda q_{s}=0 \text { for } \kappa \ll \tau .
$$

Thus we can introduce a potential $V(s, u)$. In this prototypical case, $V(s, u)=$ $\lambda q_{s}$. We can manipulate $\lambda$ to get a slightly more general potential $V(s, u)$. Thus, we obtain $i q_{u}+q_{s s}+V(s, u)=0$.

Finally, following Balakrishnan and Dandoloff [2], we present an intimate connection with the potential function $V(s, u)$ and the Fermi-Walker phase. It is clear from (2.5) and (2.7) with their Darboux vectors that if we work in a FermiWalker frame, $\mathbf{t}$ essentially gets parallel transported and the triad undergoes a rotation $\tau d s$ about $\mathbf{t}$. Similarly, the triad undergoes a rotation $h d u$ as one moves along the temporal curve by an interval $d u$. 
DEFINITION 3.2. The Fermi-Walker phase associated with an evolution of a curve undergoing a rotation around $\mathbf{t}$ is given by an angle

$$
\delta \phi^{\mathrm{FW}}=\left(\tau_{u}-h_{s}\right) d s d u .
$$

Proposition 3.3. If the evolution of the curve satisfies Heisenberg spin chain equation, then the Fermi-Walker phase satisfies

$$
\phi^{\mathrm{FW}}=V_{s} .
$$

Proof. Here $f$ and $g$ satisfy

$$
f=-\kappa \tau, \quad g=\kappa_{s} .
$$

Substituting these in equation (2.9) gives

$$
h=\left[\frac{\kappa_{s s}}{\kappa}-\tau^{2}\right] .
$$

If we preform a Hasimoto transformation to $i q_{s}+q_{s s}+V(s, u)=0$, the real part yields

$$
\tau_{s}=\left[\frac{\kappa_{s s}}{\kappa}-\tau^{2}\right]+V_{s},
$$

and our result immediately follows from the above.

Thus, in this paper, we formulated a direct and more geometrical connection between an evolution of a space curve satisfying the Heisenberg spin chain and quantum mechanics.

ACKNOwLedgments. The author is extremely grateful to Professor Partha Ghose for drawing his attention to Valery Dmitriyev's paper. He is also grateful to Professor Sarbarish Chakravarty for many helpful remarks.

\section{REFERENCES}

[1] R. Balakrishnan, A. R. Bishop, and R. Dandoloff, Geometric phase in the classical continuous antiferromagnetic Heisenberg spin chain, Phys. Rev. Lett. 64 (1990), no. 18, 2107-2110.

[2] R. Balakrishnan and R. Dandoloff, The Schrödinger equation as a moving curve, Phys. Lett. A 260 (1999), no. 1-2, 62-67.

[3] R. Balakrishnan and P. Guha, Surface-embeddability approach to the dynamics of the inhomogeneous Heisenberg spin chain, J. Math. Phys. 37 (1996), no. 8, 3651-3661.

[4] V. P. Dmitriyev, Mechanical analogy for the wave-particle: helix on a vortex filament, J. Appl. Math. 2 (2002), no. 5, 241-263.

[5] L. P. Eisenhart, An introduction to differential geometry, Princeton University Press, New Jersey, 1947.

[6] R. E. Goldstein and D. M. Petrich, The Korteweg-de Vries hierarchy as dynamics of closed curves in the plane, Phys. Rev. Lett. 67 (1991), no. 23, 3203-3206. 
[7] P. Guha, Moving space curve equations and a family of coupled KdV type systems, Chaos Solitons Fractals 15 (2003), no. 1, 41-46.

[8] H. Hasimoto, A soliton on a vortex filament, J. Fluid Mech. 51 (1972), 477-485.

[9] G. L. Lamb Jr., Solitons on moving space curves, J. Math. Phys. 18 (1977), no. 8, 1654-1661.

[10] J. Langer and R. Perline, The planar filament equation, Mechanics Day (Waterloo, Ont 1992), Fields Inst. Commun., vol. 7, American Mathematical Society, Rhode Island, 1996, pp. 171-180.

[11] K. Nakayama, H. Segur, and M. Wadati, Integrability and the motion of curves, Phys. Rev. Lett. 69 (1992), no. 18, 2603-2606.

[12] K. Nakayama and M. Wadati, Motion of curves in the plane, J. Phys. Soc. Japan 62 (1993), no. 2, 473-479.

Partha Guha: S. N. Bose National Centre for Basic Sciences, JD Block, Sector III, Salt Lake City, Calcutta 700098, India

E-mail address: guha@boson.bose.res.in

Current address: Department of Mathematics, University of Colorado at Colorado Springs, 1420 Austin Bluffs Parkway, Colorado Springs, CO 80933-7150, USA

E-mail address: pguha@math.uccs . edu 


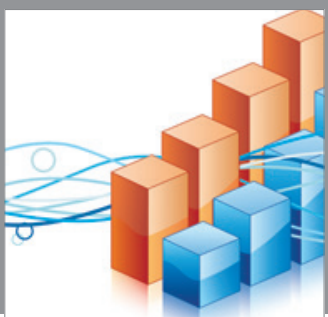

Advances in

Operations Research

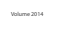

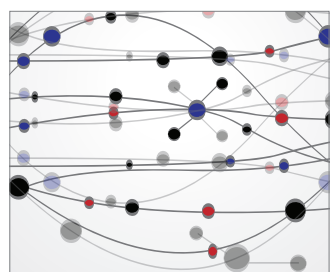

\section{The Scientific} World Journal
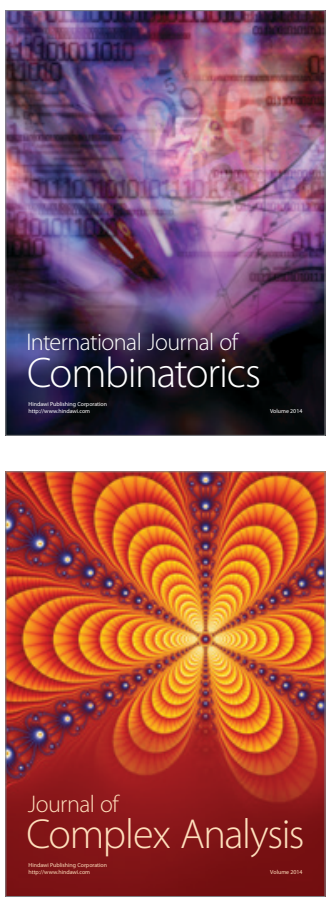

International Journal of

Mathematics and

Mathematical

Sciences
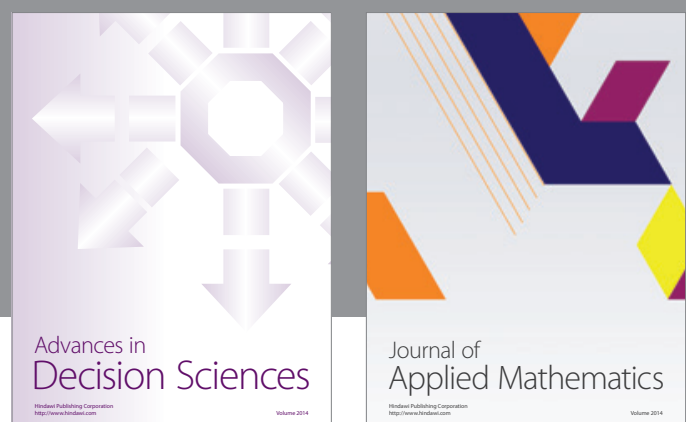

Journal of

Applied Mathematics
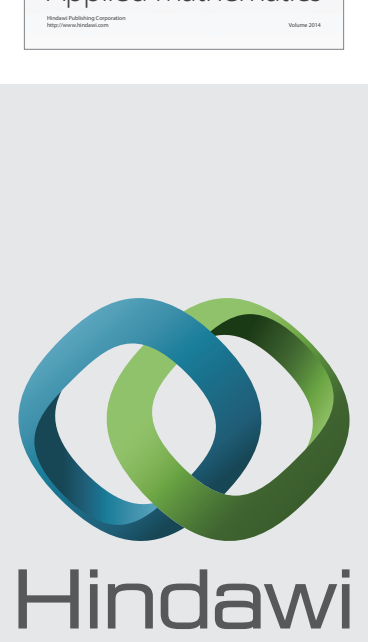

Submit your manuscripts at http://www.hindawi.com
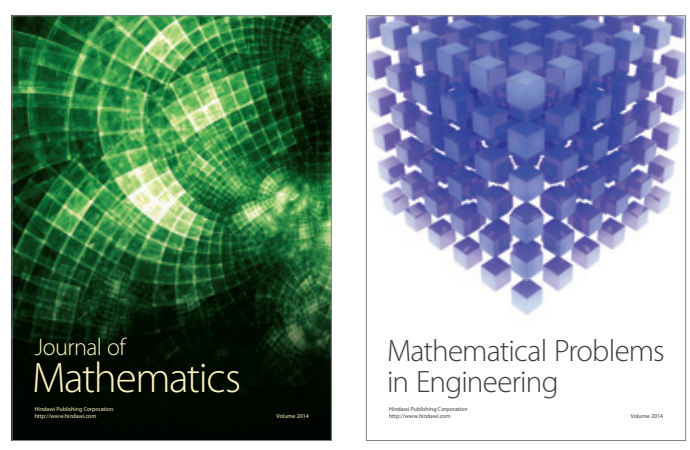

Mathematical Problems in Engineering
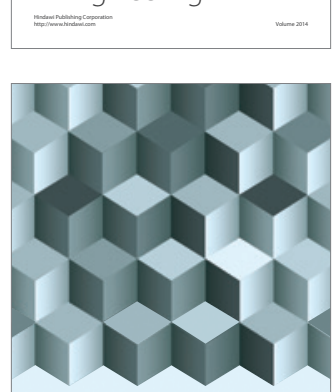

Journal of

Function Spaces
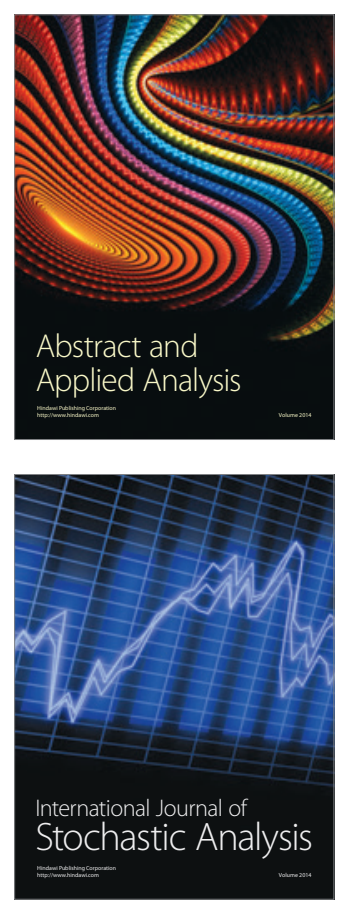

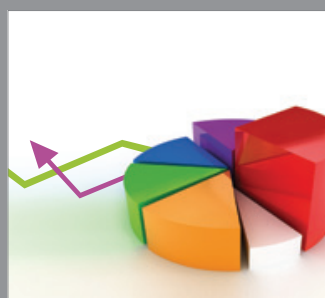

ournal of

Probability and Statistics

Promensencen
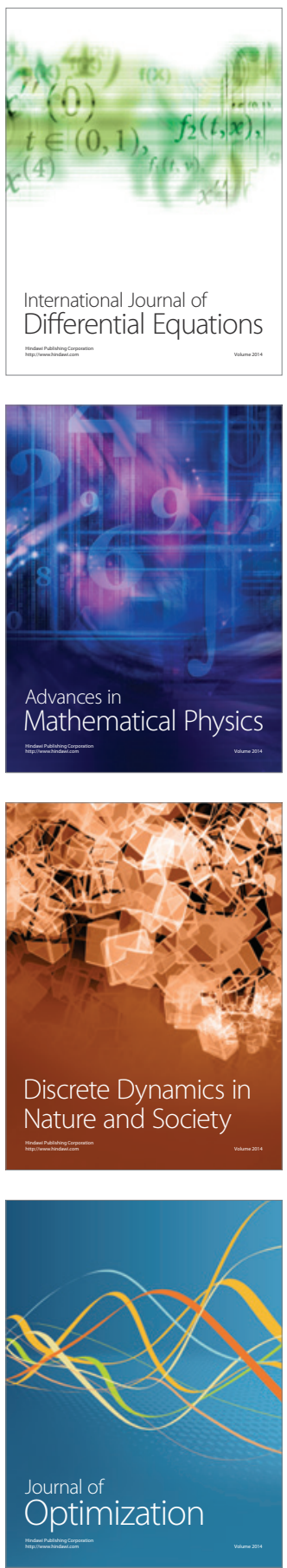